OPEN ACCESS

Edited by:

Galila Agam

Ben-Gurion University of the Negev,

Israel

Reviewed by:

Masaaki Torii,

Children's National Health System,

United States

Willayat Yousuf Wani,

University of Alabama at Birmingham,

United States

*Correspondence:

Irina Burd

iburd@jhmi.edu

Received: 31 January 2018 Accepted: 13 April 2018

Published: 03 May 2018

Citation:

Lei J, Calvo P, Vigh R and Burd I (2018) Journey to the Center of the Fetal Brain: Environmental Exposures and Autophagy.

Front. Cell. Neurosci. 12:118. doi: 10.3389/fncel.2018.00118

\section{Journey to the Center of the Fetal Brain: Environmental Exposures and Autophagy}

\author{
Jun Lei, Pilar Calvo, Richard Vigh and Irina Burd*
}

Department of Gynecology and Obstetrics, Integrated Research Center for Fetal Medicine, Johns Hopkins University School of Medicine, Baltimore, MD, United States

Fetal brain development is known to be affected by adverse environmental exposures during pregnancy, including infection, inflammation, hypoxia, alcohol, starvation, and toxins. These exposures are thought to alter autophagy activity in the fetal brain, leading to adverse perinatal outcomes, such as cognitive and sensorimotor deficits. This review introduces the physiologic autophagy pathways in the fetal brain. Next, methods to detect and monitor fetal brain autophagy activity are outlined. An additional discussion explores possible mechanisms by which environmental exposures during pregnancy alter fetal brain autophagy activity. In the final section, a correlation of fetal autophagy activity with the observed postnatal phenotype is attempted. Our main purpose is to provide the current understanding or a lack thereof mechanisms on autophagy, underlying the fetal brain injury exposed to environmental insults.

Keywords: fetal brain development, adverse perinatal neurologic outcomes, pregnancy environmental exposures, alcohol, autophagy biomarkers

\section{PHYSIOLOGY}

Autophagy is the cellular "self-eating" process by which damaged intracellular proteins, organelles and pathogens are degraded (Carloni et al., 2008; Kadandale and Kiger, 2010). Under physiologic conditions, it provides a degrade and recycle mechanism that releases amino acids, free fatty acids, and monosaccharides for reuse (Zhang F. et al., 2016). Autophagy is a highly conserved pathway common among disparate cladistic classes such as yeast, roundworms, and humans (Levine and Klionsky, 2004). Depending on the pathology, autophagy can offer a beneficial cell salvage pathway. Conversely, it can also act with apoptosis to promote cell death (Bildirici et al., 2012), especially when autophagy activity is extremely elevated (Guha et al., 2016). Autophagy may also inhibit apoptosis by way of mitochondria sequestration (Rocha-Ferreira and Hristova, 2016).

In mammals, there are three types of autophagy: macroautophagy, microautophagy, and chaperone mediated autophagy (Tekirdag and Cuervo, 2018). Macroautophagy involves the synthesis of multilayered vesicles called autophagosomes, which surround intracellular organelles such as mitochondria as well as proteins. The loaded autophagosome fuses with a lysosome, releasing lysosomal proteolytic enzymes that digest the contents of the vesicle (Hamasaki et al., 2013). Microautophagy is a similar process that does not use autophagosome vacuolates. Instead, microautophagy relies on the lysosome invaginating itself to surround and then digest the degradation target (Marzella et al., 1981; Hamasaki et al., 2013). In contrast, the targets of chaperone-mediated autophagy are not surrounded by vesicular structures. This latter process relies on chaperone proteins selecting and marking intracellular proteins which are then 
translocated across the lysosomal membrane and degraded (Kaur and Debnath, 2015). Of the three types, the most commonly studied and the one forming the basis of this review is macroautophagy, hereon referred to as autophagy.

Autophagy is a strictly controlled process mediated by many proteins (Mizushima et al., 2010; Meschini et al., 2011; Yoshii and Mizushima, 2017). An overview of the main functional protein complexes and their interactions is provided in Figure 1. Autophagy can be initiated in two primary ways. The first is via activation of AMPK (adenosine monophosphate activated protein kinase). The second is via inhibition of the nutrient sensing system mammalian target of rapamycin (mTOR) (Roos et al., 2009). Both mechanisms lead to phosphorylation and activation of the Unc-51-like kinase (ULK1) complex that starts inducting formation of the multilamellear phagophore. The ULK1 complex activates the phosphatidylinositol-3 kinase class III (PI3K CIII) complex composed of beclin-1, autophagy-related protein (ATG) 14, vacuolar protein sorting (VPS) proteins Vps34 and Vps15, which in turn generates phosphatidylinositol 3phosphate (PI3P) to facilitate membrane elongation. Various Atg proteins join together to form the Atg5-Atg12-Atg16 complex. This complex triggers the cleavage of pro-microtubule-associated protein 1 light chain 3 (LC3) to form LC3-I which is then conjugated to phosphatidylethanolamine (PE) to form LC3-II.
LC3-II promotes closure of the vesicle membrane, which is the event that signals the final step in autophagosome vacuole formation.

The importance of autophagy is evident early in human embryonic development. During the late two cell zygotic stage, autophagy is actively degrading maternally derived proteins that originated from the oocyte but are now exhausted (Nakashima et al., 2017). The low oxygen tension of the endometrial cavity forces the blastocyst to induce autophagy to achieve extravillous trobphoblast invasion (Genbacev et al., 1997). Subsequent LC3 activity in early pregnancy is also diffuse and ubiquitous (Avagliano et al., 2016), as characterized by the extensive neural tube defects observed when the Ambral protein in the PI3K-III complex is absent (Fimia et al., 2007), as well as by strong LC3 signaling in the structures formed by neural crest cells (Cann et al., 2008). Autophagy is also highly active and necessary for neuronal differentiation (Zhao et al., 2010; Avagliano et al., 2016). Experiments have also shown that Atg5 (Mizushima et al., 2001; Kuma et al., 2004; Klionsky et al., 2016) or Atg7 (Komatsu et al., 2005) deletion is characterized by early neonatal death. At its most basic level, autophagy is basally present as an ongoing constituitive process in every cell, though new evidence suggests that different cell types regulate autophagy distinctly (Nakashima et al., 2017).

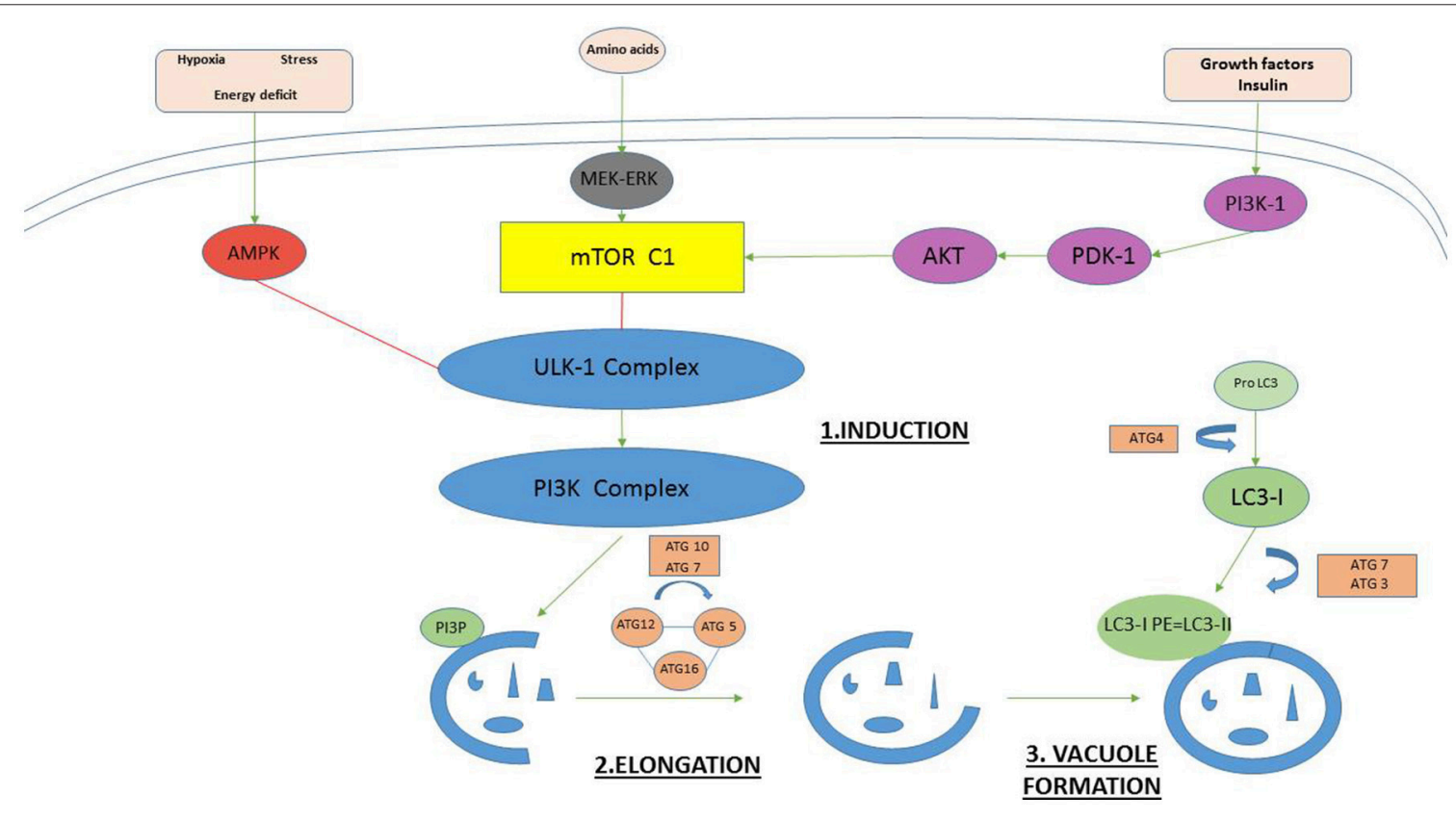

FIGURE 1 | The key proteins in mammalian autophagosome formation. Autophagy can be initiated in two primary ways. The first is via activation of AMPK (adenosine monophosphate activated protein kinase) under hypoxia, stress, and energy deficit. The second is via inhibition of the nutrient sensing system mammalian target of rapamycin (mTOR). Both mechanisms lead to phosphorylation and activation of the Unc-51-like kinase (ULK1) complex that starts inducting formation of the multilamellear phagophore. The ULK1 complex activates the phosphatidylinositol-3 kinase class III (PI3K CIII) complex composed of beclin-1, Atg14, vacuolar protein sorting (VPS) proteins Vps34 and Vps15, which in turn generates phosphatidylinositol 3-phosphate (PI3P) to facilitate membrane elongation. Various Atg proteins join together to form the Atg5-Atg12-Atg16 complex. This complex triggers the cleavage of pro-microtubule-associated protein 1 light chain 3 (LC3) to form LC3-I which is then conjugated to phosphatidylethanolamine (PE) to form LC3-II. LC3-II promotes closure of the vesicle membrane, which is the event that signals the final step in autophagosome vacuole formation. 


\section{DETECTING ALTERED FETAL BRAIN AUTOPHAGY ACTIVITY}

It is essential to demonstrate and measure physiologic autophagy first before discussing pathologic autophagy. In commenting on the problem of measuring autophagy activity, Mizushima et al. declared there is "no gold standard" (Mizushima et al., 2010; Klionsky et al., 2016). This is likely due to the myriad of techniques available, including immunohistochemistry, immunoblotting (Jiang and Mizushima, 2015), fluorescence microscopy, electron microscopy, radiolabeling, flow cytometry, and fluorescent probes (Cui et al., 2017; Yoshii and Mizushima, 2017). The autophagy factors most commonly used to measure bilaminar membrane formation and elongation include ULK1, WIPI1/2 [tryptophan (W), aspartic acid (D) repeat domain phosphoinositide-interacting protein], Atg5, and LC3. However, timing when to perform these techniques requires careful planning as autophagic flux may obscure overall trends in autophagy. Measurements of autophagy activity should quantitatively capture its presence or absence. Ideally, measurements should also be recorded over time, so as to reveal trends in the rate of phagosome formation and autophagolysosome degradation. Such intermittent measurement of autophagy activity can pose a challenge. Direct visualization of autophagy vesicles requires electron or immunofluorescence microscopy. Either method requires a cumbersome tissue preparation and image acquisition process that can make the task of creating multiple snapshots to trend autophagosome formation and fusion onerous. Consequently, it is desirable to have insight into the opportune time to perform a measurement so as to capture autophagy activity when it is occurring. For example, Fineschi suggested a model of hypoxia that first uses chaperone proteins such as heat shock proteins (HSPs) and oxygen-regulated protein (ORP150) to detect the onset of the inflammatory response to hypoxia (Fineschi et al., 2017).

The single most common measured autophagy marker is LC3. Avagliano et al. assessed autophagy activity distribution and intensity during development of neural tissue in mouse embryos and human fetuses. Their work revealed similar spatiotemporal autophagy trends using immunofluorescence to detect LC3 expression. One hazard of LC3 measurement lies in that LC3 can be elevated in the context of authophagosome degradation inhibition as well as in ectopy. Autophagy activity can also be in a state of flux. Accordingly, one must measure the change in LC3II over time (Yoshii and Mizushima, 2017). Co-measurement of LC3-II with degradation of p62 can be used as the latter is directly attached to LC3 and degrades with autophagy (Bjørkøy et al., 2005; Mizushima and Hara, 2006; Pankiv et al., 2007), though p62 should also be used with caution in the setting of starvation ( $>2 \mathrm{~h}$ ) (Jiang and Mizushima, 2015). Alternatively, measuring LC3-II in the presence and absence of autophagy inhibitors such as bafilomycin can be used to increase the accuracy of LC3-II flux measurement (Yoshii and Mizushima, 2017). Radiolabeling amino acids inside cells and then incubating the cells for a time that is long enough to allow proteins with short half-lives to turn over but short enough so as to prevent the reincorporation of freed amino acids into new proteins again facilitates measurement of autphagy flux (Yoshii and Mizushima, 2017).

In another approach to measuring autophagy activity, a tandem fluorescent protein quenching assay joins together RFP (red fluorescent protein), GFP (green fluorescence protein), and LC3 into a single RFP-GFP-LC3B tag (Thermo Fisher Scientific: Waltham, MA). The fluorescence of the tag can be exploited to expose acidic compartments, such as those found in lysosomes. While the green GFP LC3-II and red RFP fluorescence tags together shine yellow and are both present in the neutral $\mathrm{pH}$ of the autophagosome, the green GFP LC3-II signal is quenched in the acidic environment of the lysosome, leaving the $\mathrm{pH}$-stable mRFP-LC3 signal to fluoresce red alone (Kimura et al., 2007).

Shvets et al. showed how flow cytometry could be used to detect the levels of fluorescence proteins. The decrease in GFPLC3 fluorescence reflects ongoing autophagy activity (Shvets et al., 2008).

Each autophagic assay has its own limitations. Ultrastructural analysis (TEM) is insufficient to deal with the biological variability and heterogeneity of an organ/tissue, which requires specialized expertise. Fluorescent microscope or flow cytometry has the potential experimental pitfalls, such as potential for subjectivity, uniform, and criteria for quantitation. Immunoblotting could be less sensitive and informative when analyzing tissue- or stage-specific variations. As autophagy involves dynamic and complicated processes, it is very important to carry out proper assays that deal with the nature of autophagy. All tests that are listed in the Table $\mathbf{1}$ as well.

\section{DISCUSSION OF ENVIRONMENTAL EXPOSURES}

Multiple different environmental exposures alter autophagy activity in the fetal brain (Table 1). Broadly, many of them can be characterized as infectious, hypoxic, and toxic. While the mechanism detailing the pathway from affect to phenotype is urgently needed, at least some evidence shows that autophagy influences these phenotypic presentations.

Multiple infectious agents alter autophagy as part of their pathogenic exertion. The Zika virus (ZIKV) may cross the placental barrier via a special type of autophagy called secretory autophagy (Zhang Z. W. et al., 2016). Once crossed, the virus hones in on tropic factors (Miner and Diamond, 2017) to infect human fetal neural stem cells (fNSCs). Once inside an fNSC, ZIKV makes NS4a and NS4b proteins that decrease mTOR and induce autophagy (Liang et al., 2016) in a way that impairs neurogenesis (Chiramel and Best, 2017). ZIKV also causes microcephaly via apoptosis and autophagy-driven cell death of cortical progenitor cells (Cugola et al., 2016). Research has shown that treatment with the autophagy inhibitor hydroxychloroquine reduces the incidence of ZIKV vertical transmission in pregnancy (Cao et al., 2017).

Similar to Zika, HIV also exerts its some of its effects via autophagy. Once HIV has infected an astrocyte, it produces 


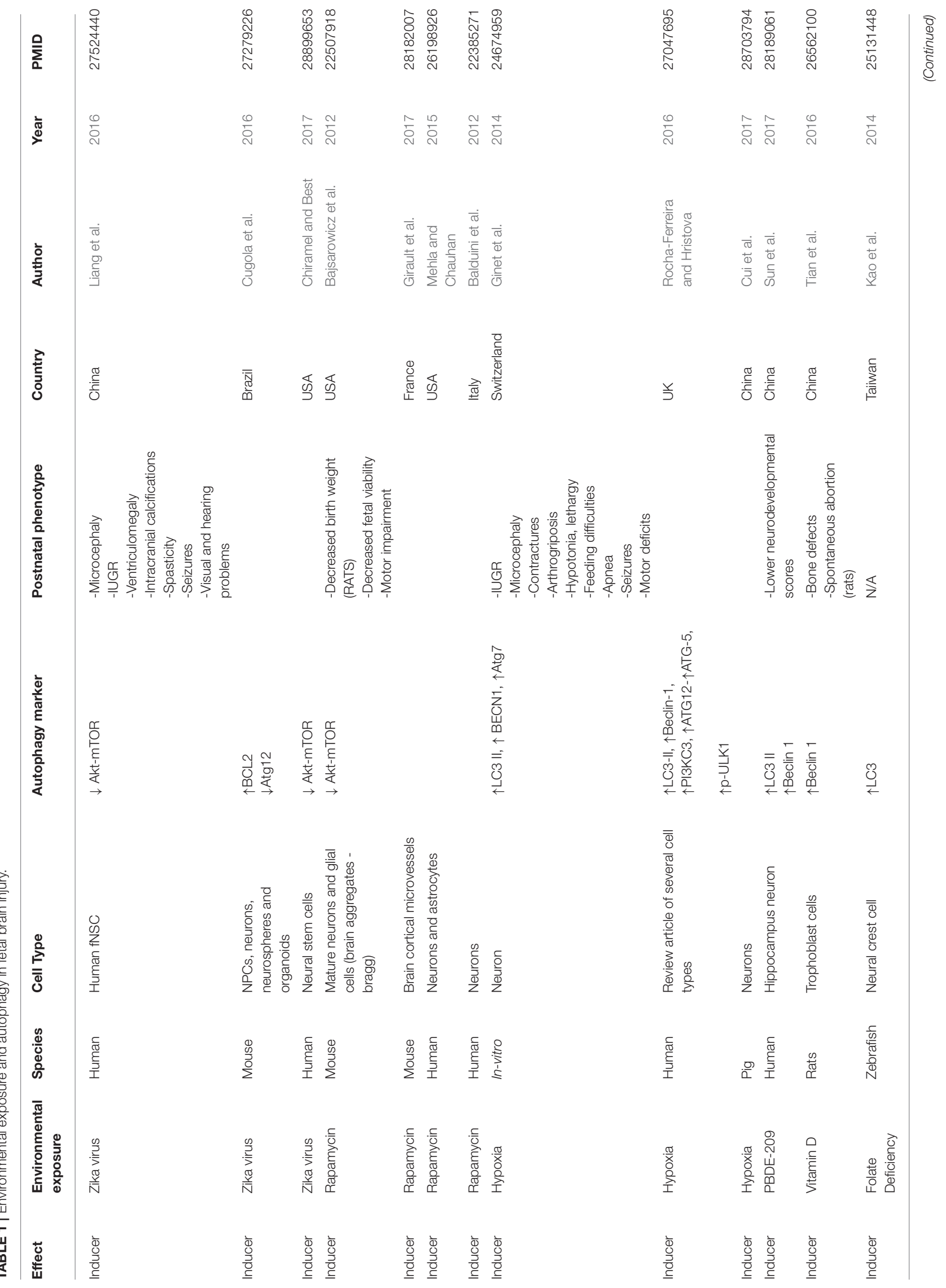




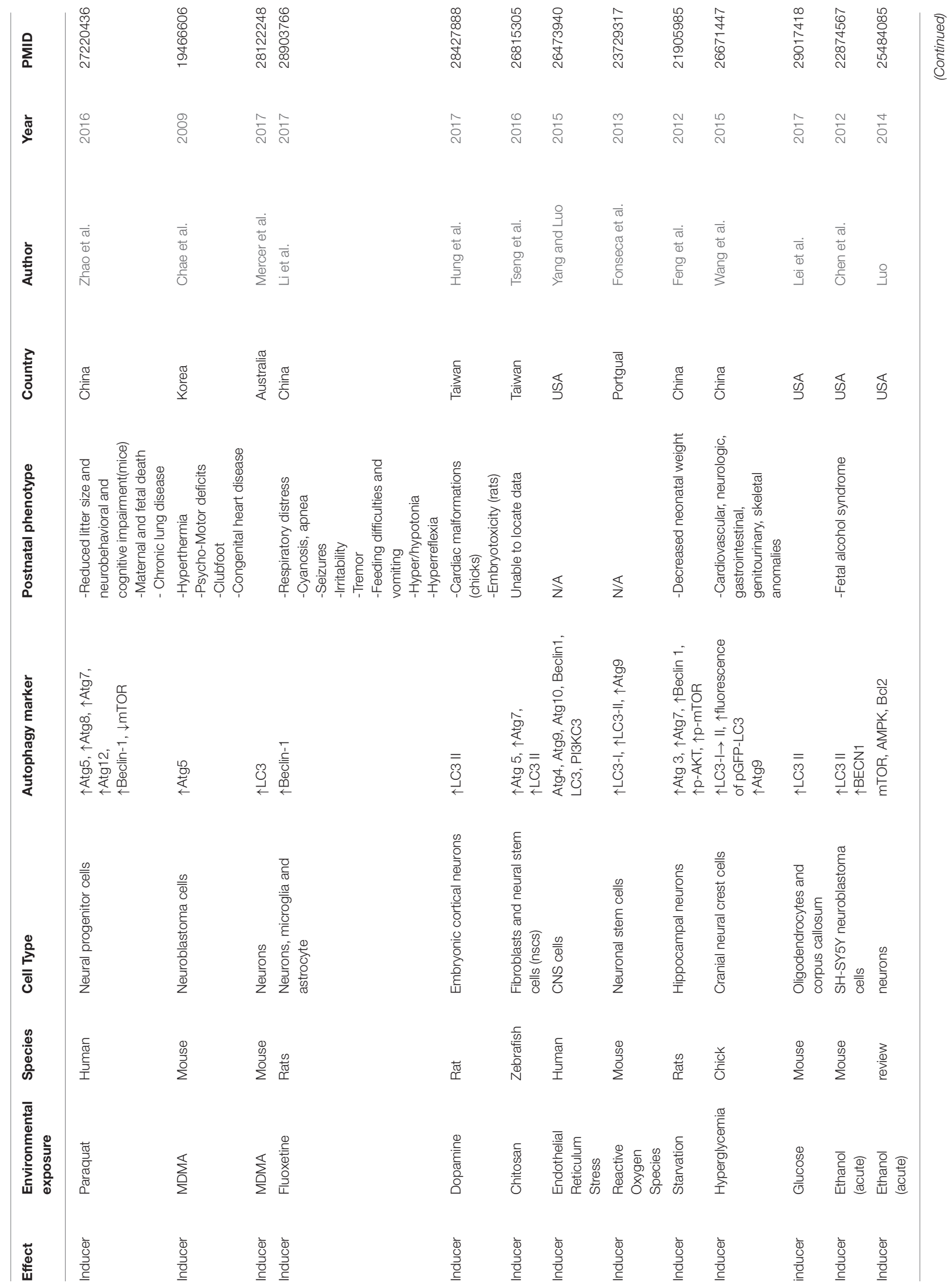




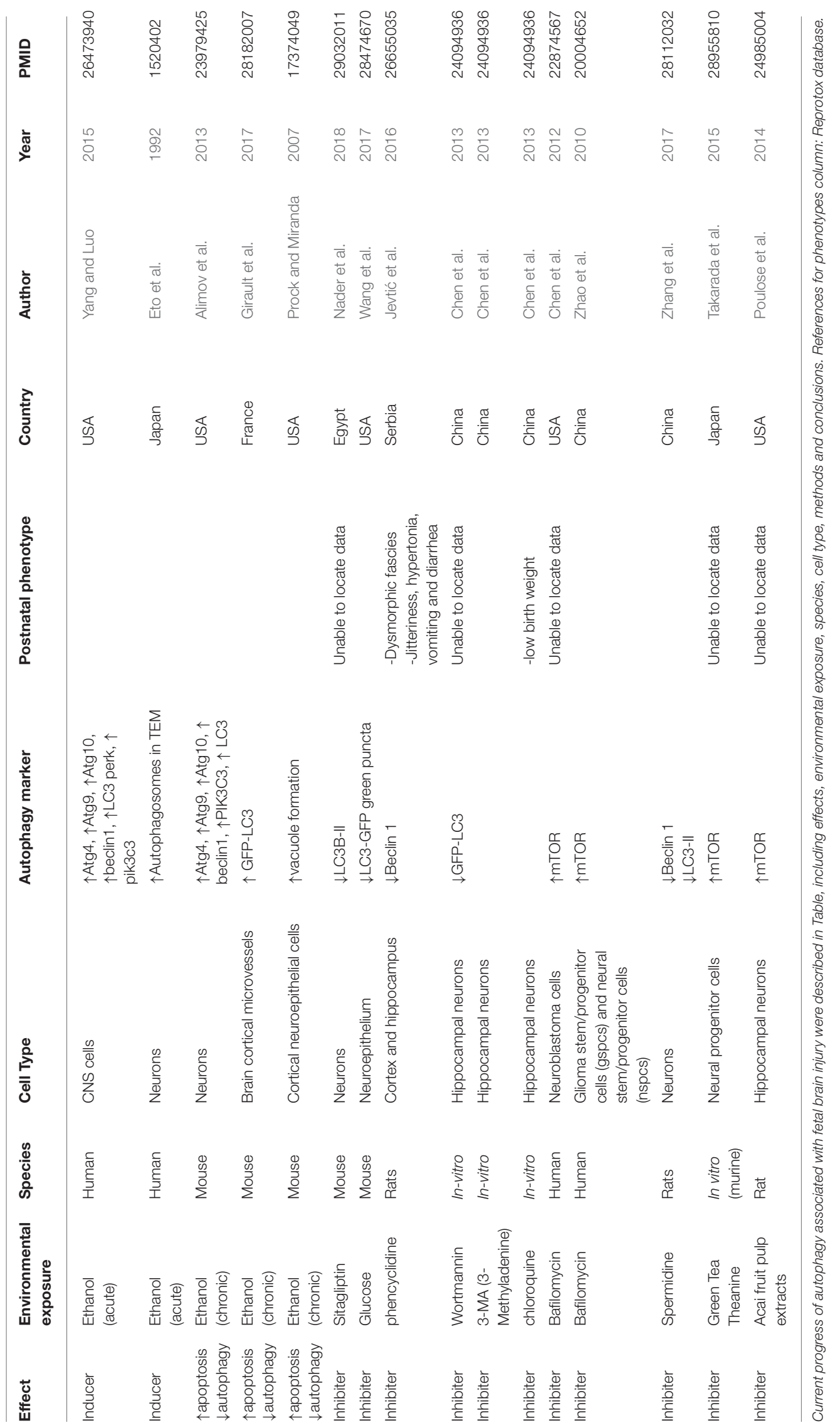


a Nef protein that is associated with the HIV-Associated Neurocognitive Disorders (HAND) phenotype. It has been shown that infecting fetal astrocytes with an adenovirus based vector of the Nef protein leads to accumulation of autophagosomes by way of blocking their fusion with lysosomes. As autophagy has an essential role in innate immunity, the manufacture of Nef by HIV safeguards its presence inside the cell and allows it to avoid destruction by the lysosome's enzymatic and acidic environment (Saribas et al., 2015).

While hypoxia and infection may be the result of different underlying etiologies, they share a common disease pathway in autophagy. The exact role of autophagy in neonatal hypoxicischemic encephalopathy (HIE) is controversial. The findings in several rodent studies have demonstrated conflicting roles for autophagy, with some data suggesting a cell-protective role (Carloni et al., 2008; Wang et al., 2012) while other studies suggest autophagy led to a cell-death pathway (Koike et al., 2008; Wen et al., 2008; Bidlingmaier et al., 2009; Xing et al., 2012). Moving away from the rodent model, Ginet et al. has shown in late pre-term and term neonates that HIE increased the number of autophagosomes and lysosomes by one order of magnitude in the asphyxia-sensitive ventrolateral thalamic region of the brain. They demonstrated comparable findings in both rodent and human neonates who died after acute perinatal HIE. Their collected biomarker data also demonstrated increased autophagy activity with significant increases in LC3-II activity and simultaneous decreases in p62 (Ginet et al., 2014).

In addition to the autophagy mediated hazards posed by infection and hypoxia, toxins also potently influence autophagy. For example, exposure to ethanol during the ethanol sensitive period of pregnancy leads to an increase in apoptosis and a decrease in autophagy. Earlier research in humans suggested that the ethanol sensitive period of pregnancy was as late as 20 weeks of gestation. More recent data from a rodent model shows less autophagy activity and increased apoptotic markers when mice are exposed to ethanol on post natal day 4, a time period which approximately correlates to the third trimester in humans. Conversely, later in pregnancy, when the fetal brain stress response system has matured, ethanol exposure leads to an increase in multiple autophagy markers that are accompanied by significantly fewer apoptotic markers, signaling the onset of an ethanol resistant period in pregnancy (Alimov et al., 2013).

In addition to gestational timing, ethanol exposure has a second temporal affect on autophagy in the fetal brain. Acute ethanol exposure induces autophagy activity to protect the developing brain. In contrast, chronic ethanol exposure in adult progeny activates mTOR, thereby inhibiting the autophagy pathway in the brain (24556681). Consequently, chronic ethanol exposure may impair protective autophagy function when fetal neurons are faced with increased stress.

A second toxin associated with recreational drug use is 3,4-methylenedioxymethamphetamine (MDMA). It is also used to treat post traumatic stress disorder (PTSD) (Amoroso and Workman, 2016). MDMA has been shown to upregulate autophagy in the fetal brain by increasing Atg5 and LC3 levels (Chae et al., 2009). Consuming MDMA during pregnancy is related to fetal neural and cardiotoxicity as well as impaired motor functioning (Meamar et al., 2010).

Though now less commonly abused, phencyclidine (PCP) acts as a non-competitive antagonist of the glutamatergic $\mathrm{N}$ methyl-d-aspartate (NMDA) receptor. Its administration to pregnant rats alters the behavior of their offspring and causes neurodegenerative effects similar to those seen in schizophrenia (SCH) (Radonjić et al., 2008). Autophagy has also been shown to play a key role in the disease mechanism of SCH (MerenlenderWagner et al., 2015). A recent study shows the presence of autophagy in a PCP model of SCH in rodents. After PCP administration, autophagy downregulation was seen by way of reduced Beclin1 expression in the neocortex and in the hippocampus (Jevtić et al., 2016).

While some maternal exposures are part of the spectrum of substance abuse, others are so ubiquitous that they are difficult to avoid. For example, paraquat is a commonly used herbicide. Paraquat exposure to a human progenitor cell line is associated with an increase in autophagy (Zhao et al., 2016). In the adult phenotype, paraquat has been linked to Parkinson's disease in farm workers through an increased production of reactive oxygen species exerting their toxicity on neurons (Tanner et al., 2011).

A final category of substances that alter autophagy brain activity in cells is very small metal particles. Metals, due to their nature, cannot be degraded by a lysosome's enzymatic complement. Consequently, the entry of micronized metals into a cell may induce autophagy though the digestive process cannot be completed. Instead, the particles accumulate in autophagosomes. Researchers have raised concerns about the impact the fetal and neonatal brain may suffer from altered autophagy activity from exposure to silver (Guo et al., 2017) and titanium dioxide nanoparticles (Song et al., 2016), as well as even smaller Cadmium Selenide / Zinc Sulfide (CdSe/ZnS) quantum dots (Chen et al., 2013).

The previous discussions on various exposures are not exhaustive and we cannot explain the mechanism for all the exposures and phenotypes listed in Table 1. However, we can reasonably speculate that autophagy plays at least some role in certain phenotypic presentations. For example, progeny with features of decreased physical size, such as microcephaly, low birth weight, or IUGR may be attributable to elevated autophagy levels. The psycho-sensorimotor deficits seen with certain exposures may also be attributable to dysregulation of autophagy activity in neurons.

\section{FUTURE INVESTIGATIONS}

All markers of autophagy activity cited in Table 1 were recovered post-mortem or in-vitro. Future research is urgently needed to employ these markers for in utero identification of potential disease processes associated with environmental exposures in pregnancy.

While many environmental exposures that influence autophagy activity have been identified, many remain to be discovered. The discovery of additional safe and economical in vivo modulators of autophagy would offer 
additional tools to reverse common pathogenic affecters of autophagy.

Three promising treatments for altered autophagy levels include glucose, modest hypoxia, and hydroxychloroquine.

Our recent discovery of glucose infusion for pathogenic autophagy activity represents a potentially accessible and costeffective treatment of perinatal brain injury in the setting of intrauterine inflammation secondary to chorioamnionitis or preterm birth (Lei et al., 2017).

A potential treatment for chronic ethanol exposure could lie in the development of safe autophagy inducers. This has already been demonstrated in a limited way by a study which showed that a modest hypoxic preconditioning induced protective autophagy in human neuronal stem cell cultures affected by long term exposure to alcohol (Luo, 2014).

While the peak of the recent Zika outbreak in the Americas has passed, it is imperative that further research is performed

\section{REFERENCES}

Alimov, A., Wang, H., Liu, M., , Frank, J. A., Xu, M., Ou, X., et al. (2013). Expression of autophagy and UPR genes in the developing brain during ethanol-sensitive and resistant periods. Metab. Brain Dis. 28, 667-676. doi: 10.1007/s11011-013-9430-2

Amoroso, T., Workman, M. (2016). Treating posttraumatic stress disorder with MDMA-assisted psychotherapy: A preliminary meta-analysis and comparison to prolonged exposure therapy. J. Psychopharmacol. 30, 595-600. doi: 10.1177/0269881116642542

Avagliano, L., Doi, P., Tosi, D., Scagliotti, V., Gualtieri, A., Gaston-Massuet, C., et al. (2016). Cell death and cell proliferation in human spina bifida. Birth Defects Res. A Clin. Mol. Teratol. 106, 104-113. doi: 10.1002/bdra.23466

Bajsarowicz, K., Ahn, M., Ackerman, L., Dearmond, B. N., Carlson, G., and DeArmond, S. J. (2012). A brain aggregate model gives new insights into the pathobiology and treatment of prion diseases. J. Neuropathol. Exp. Neurol. 71, 449-466. doi: 10.1097/NEN.0b013e3182544680

Balduini, W., Carloni, S., and Buonocore, G. (2012). Autophagy in hypoxiaischemia induced brain injury. J. Matern. Fetal Neonatal Med. 25(Suppl. 1), 30-34. doi: 10.3109/14767058.2012.663176

Bidlingmaier, C., Eberl, W., Knöfler, R., Olivieri, M., and Kurnik, K. (2009). Haemostatic testing prior to elective surgery in children? Not always! Hamostaseologie 29, 64-67. doi: 10.1055/s-0037-1616942

Bildirici, I., Longtine, M. S., Chen, B., and Nelson, D. M. (2012). Survival by self-destruction: a role for autophagy in the placenta? Placenta 33, 591-598. doi: 10.1016/j.placenta.2012.04.011

Bjørkøy, G., Lamark, T., Brech, A., Outzen, H., Perander, M., Overvatn, A., et al. (2005). p62/SQSTM1 forms protein aggregates degraded by autophagy and has a protective effect on huntingtin-induced cell death. J. Cell Biol. 171, 603-614. doi: $10.1083 /$ jcb.200507002

Cann, G. M., Guignabert, C., Ying, L., Deshpande, N., Bekker, J. M., Wang, L., et al. (2008). Developmental expression of LC3alpha and beta: absence of fibronectin or autophagy phenotype in LC3beta knockout mice. Dev. Dyn. 237, 187-195. doi: 10.1002/dvdy.21392

Cao, B., Parnell, L. A., Diamond, M. S., and Mysorekar, I. U. (2017). Inhibition of autophagy limits vertical transmission of Zika virus in pregnant mice. J. Exp. Med. 214, 2303-2313. doi: 10.1084/jem.20170957

Carloni, S., Buonocore, G., and Balduini, W. (2008). Protective role of autophagy in neonatal hypoxia-ischemia induced brain injury. Neurobiol. Dis. 32, 329-339. doi: 10.1016/j.nbd.2008.07.022

Chae, M., Rhee, G. S., Jang, I. S., Kim, K., Lee, J. H., Lee, S. Y., et al. (2009). ATG5 expression induced by MDMA (ecstasy), interferes with neuronal differentiation of neuroblastoma cells. Mol. Cells 27, 571-575. doi: 10.1007/s10059-009-0075-2 about its management. Attempting to affect its clinical course with the use of autophagy modulating molecules such as hydroxychloroquine or possible acai derivatives offers new areas for research.

Finally, further studies are needed in order to elucidate the causal connections between altered embryonic and fetal autophagy activity and psychiatric disorders.

\section{AUTHOR CONTRIBUTIONS}

IB: Designed review and selected articles for the synopsis, guided manuscript structure, and supervised writing: PC: Reviewed literature and participated in the writing of the manuscript; $\mathrm{RV}$ : Reviewed literature and participated in the writing of the manuscript; JL: Helped in article selection for the review, reviewed literature, and participated in the writing of the manuscript.
Chen, G., Ke, Z., Xu, M., Liao, M., Wang, X., Qi, Y., et al. (2012). Autophagy is a protective response to ethanol neurotoxicity. Autophagy 8, 1577-1589. doi: 10.4161/auto. 21376

Chen, L., Miao, Y., Jin, P., Zha, Y., Chai, Y., Zheng, F., et al. (2013). The role of elevated autophagy on the synaptic plasticity impairment caused by CdSe/ZnS quantum dots. Biomaterials 34, 10172-10181. doi: 10.1016/j.biomaterials.2013.09.048

Chiramel, A. I., and Best, S. M. (2017). Role of autophagy in Zika virus infection and pathogenesis. Virus Res. doi: 10.1016/j.virusres.2017.09.006. [Epub ahead of print].

Cugola, F. R., Fernandes, I. R., Russo, F. B., Freitas, B. C., Dias, J. L., Guimarães, K. P., et al. (2016). The Brazilian Zika virus strain causes birth defects in experimental models. Nature 534, 267-271. doi: 10.1038/nature18296

Cui, D., Sun, D., Wang, X., Yi, L., Kulikowicz, E., Reyes, M., et al. (2017). Impaired autophagosome clearance contributes to neuronal death in a piglet model of neonatal hypoxic-ischemic encephalopathy. Cell Death Dis. 8:e2919. doi: $10.1038 /$ cddis.2017.318

Eto, K., Oyanagi, S., Itai, Y., Tokunaga, H., Takizawa, Y., and Suda, I. (1992). A fetal type of Minamata disease. An autopsy case report with special reference to the nervous system. Mol. Chem. Neuropathol. 16, 171-186.

Feng, Z., Zou, X., Jia, H., Li, X., Zhu, Z., Liu, X., et al. (2012). Maternal docosahexaenoic acid feeding protects against impairment of learning and memory and oxidative stress in prenatally stressed rats: possible role of neuronal mitochondria metabolism. Antioxid. Redox Signal. 16, 275-289. doi: 10.1089 /ars.2010.3750

Fimia, G. M., Stoykova, A., Romagnoli, A., Giunta, L., Di Bartolomeo, S., Nardacci, R., et al. (2007). Ambral regulates autophagy and development of the nervous system. Nature 447, 1121-1125. doi: 10.1038/nature05925

Fineschi, V., Viola, R. V., La Russa, R., Santurro, A., and Frati, P. (2017). A controversial medicolegal issue: timing the onset of perinatal hypoxic-ischemic brain injury. Mediators Inflamm. 2017:6024959. doi: 10.1155/2017/6024959

Fonseca, M. B., Sola, S., Xavier, J. M., Dionisio, P. A., and Rodrigues, C. M. (2013). Amyloid $\beta$ peptides promote autophagy-dependent differentiation of mouse neural stem cells: A $\beta$-mediated neural differentiation. Mol. Neurobiol. 48, 829-840. doi: 10.1007/s12035-013-8471-1

Genbacev, O., Zhou, Y., Ludlow, J. W., and Fisher, S. J. (1997). Regulation of human placental development by oxygen tension. Science 277, 1669-1672. doi: $10.1126 /$ science.277.5332.1669

Ginet, V., Pittet, M. P., Rummel, C., Osterheld, M. C., Meuli, R., Clarke, P. G.,et al. (2014). Dying neurons in thalamus of asphyxiated term newborns and rats are autophagic. Ann. Neurol. 76, 695-711. doi: 10.1002/ana.24257

Girault, V., Gilard, V., Marguet, F., Lesueur, C., Hauchecorne, M., Ramdani, Y., et al. (2017). Prenatal alcohol exposure impairs autophagy in neonatal brain cortical microvessels. Cell Death Dis. 8:e2610. doi: 10.1038/cddis.2017.29 
Guha, P., Harraz, M. M., and Snyder, S. H. (2016). Cocaine elicits autophagic cytotoxicity via a nitric oxide-GAPDH signaling cascade. Proc. Natl. Acad. Sci. U.S.A. 113, 1417-1422. doi: 10.1073/pnas. 1524860113

Guo, X., Zhang, G., Chen, L., Khan, A. A., Gu, B., and Li, B. (2017). Newborn neurons are damaged in vitro by a low concentration of silver nanoparticles through the inflammatory oxidative stress pathway. DNA Cell Biol. 36, 1062-1070. doi: 10.1089/dna.2017.3795

Hamasaki, M., Shibutani, S. T., and Yoshimori, T. (2013). Up-to-date membrane biogenesis in the autophagosome formation. Curr. Opin. Cell Biol. 25, 455-460. doi: 10.1016/j.ceb.2013.03.004

Hung, H. H., Kao, L. S., Liu, P. S., Huang, C. C., Yang, D. M., and Pan, C. Y. (2017). Dopamine elevates intracellular zinc concentration in cultured rat embryonic cortical neurons through the cAMP-nitric oxide signaling cascade. Mol. Cell. Neurosci. 82, 35-45. doi: 10.1016/j.mcn.2017.04.006

Jevtić, G., Nikolić, T., Mirčić, A., Stojković, T., Velimirović, M., Trajković, V., et al. (2016). Mitochondrial impairment, apoptosis and autophagy in a rat brain as immediate and long-term effects of perinatal phencyclidine treatment - influence of restraint stress. Prog. Neuropsychopharmacol. Biol. Psychiatry 66, 87-96. doi: 10.1016/j.pnpbp.2015.11.014

Jiang, P., and Mizushima, N. (2015). LC3- and p62-based biochemical methods for the analysis of autophagy progression in mammalian cells. Methods 75, 13-18. doi: 10.1016/j.ymeth.2014.11.021

Kadandale, P., and Kiger, A. A. (2010). Role of selective autophagy in cellular remodeling: "self-eating" into shape. Autophagy 6, 1194-1195. doi: 10.4161/auto.6.8.13476

Kao, T. T., Chu, C. Y., Lee, G. H., Hsiao, T. H., Cheng, N. W., Chang, N. S., et al. (2014). Folate deficiency-induced oxidative stress contributes to neuropathy in young and aged zebrafish-implication in neural tube defects and Alzheimer's diseases. Neurobiol. Dis. 71, 234-244. doi: 10.1016/j.nbd.2014.08.004

Kaur, J., Debnath, J. (2015). Autophagy at the crossroads of catabolism and anabolism. Nat. Rev. Mol. Cell Biol. 16, 461-472. doi: 10.1038/nrm4024

Kimura, S., Noda, T., and Yoshimori, T. (2007). Dissection of the autophagosome maturation process by a novel reporter protein, tandem fluorescent-tagged LC3. Autophagy 3, 452-460. doi: 10.4161/auto.4451

Klionsky, D. J., Abdelmohsen, K., Abe, A., Abedin, M. J., Abeliovich, H., Arozena, A. A., et al. (2016). Guidelines for the use and interpretation of assays for monitoring autophagy (3rd edition). Autophagy 12, 1-222. doi: 10.1080/15548627.2015.1100356

Koike, M., Shibata, M., Tadakoshi, M., Gotoh, K., Komatsu, M., Waguri, S., et al. (2008). Inhibition of autophagy prevents hippocampal pyramidal neuron death after hypoxic-ischemic injury. Am. J. Pathol. 172, 454-469. doi: 10.2353/ajpath.2008.070876

Komatsu, M., Waguri, S., Ueno, T., Iwata, J., Murata, S., Tanida, I., et al. (2005). Impairment of starvation-induced and constitutive autophagy in Atg7deficient mice. J. Cell Biol. 169, 425-434. doi: 10.1083/jcb.200412022

Kuma, A., Hatano, M., Matsui, M., Yamamoto, A., Nakaya, H., Yoshimori, T., et al. (2004). The role of autophagy during the early neonatal starvation period. Nature 432, 1032-1036. doi: 10.1038/nature03029

Lei, J., Zhong, W., Almalki, A., Zhao, H., Arif, H., Rozzah, R., et al. (2017). Maternal glucose supplementation in a murine model of chorioamnionitis alleviates dysregulation of autophagy in fetal brain. Reprod. Sci. doi: $10.1177 / 1933719117734321$. [Epub ahead of print].

Levine, B., and Klionsky, D. J. (2004). Development by self-digestion: molecular mechanisms and biological functions of autophagy. Dev. Cell. 6, 463-477. doi: 10.1016/S1534-5807(04)00099-1

Li, J. R., Xu, H. Z., Nie, S., Peng, Y., Fan, L. F., Wang, Z., et al. (2017). Fluoxetine-enhanced autophagy ameliorates early brain injury via inhibition of NLRP3 inflammasome activation following subrachnoid hemorrhage in rats. J. Neuroinflammation 14:186. doi: 10.1186/s12974-017-0959-6

Liang, Q., Luo, Z., Zeng, J., Chen, W., Foo, S. S., Lee, S. A., et al. (2016). Zika virus NS4A and NS4B proteins deregulate Akt-mTOR signaling in human fetal neural stem cells to inhibit neurogenesis and induce autophagy. Cell Stem Cell 19, 663-671. doi: 10.1016/j.stem.2016.07.019

Luo, J. (2014). Autophagy and ethanol neurotoxicity. Autophagy 10, 2099-2108. doi: $10.4161 / 15548627.2014 .981916$

Marzella, L., Ahlberg, J., and Glaumann, H. (1981). Autophagy, heterophagy, microautophagy and crinophagy as the means for intracellular degradation. Virchows Arch. B 36, 219-234. doi: 10.1007/BF02912068
Meamar, R., Karamali, F., Sadeghi, H. M., Etebari, M., Nasr-Esfahani, M. H., and Baharvand, H. (2010). Toxicity of ecstasy (MDMA) towards embryonic stem cell-derived cardiac and neural cells. Toxicol. In Vitro 24, 1133-1138. doi: 10.1016/j.tiv.2010.03.005

Mehla, R., and Chauhan, A. (2015). HIV-1 differentially modulates autophagy in neurons and astrocytes. J. Neuroimmunol. 285, 106-118. doi: 10.1016/j.jneuroim.2015.06.001

Mercer, L. D., Higgins, G. C., Lau, C. L., Lawrence, A. J., and Beart, P. M. (2017). MDMA-induced neurotoxicity of serotonin neurons involves autophagy and rilmenidine is protective against its pathobiology. Neurochem. Int. 105, 80-90. doi: 10.1016/j.neuint.2017.01.010

Merenlender-Wagner, A., Malishkevich, A., Shemer, Z., Udawela, M., Gibbons, A., Scarr, E., et al. (2015). Autophagy has a key role in the pathophysiology of schizophrenia. Mol. Psychiatry 20, 126-132. doi: 10.1038/mp.2013.174

Meschini, S., Condello, M., Lista, P., and Arancia, G. (2011). Autophagy: Molecular mechanisms and their implications for anticancer therapies. Curr Cancer Drug Targets 11, 357-379. doi: 10.2174/156800911794519707

Miner, J. J., and Diamond, M. S. (2017). Zika virus pathogenesis and tissue tropism. Cell Host Microbe 21, 134-142. doi: 10.1016/j.chom.2017.01.004

Mizushima, N., and Hara, T. (2006). Intracellular quality control by autophagy: how does autophagy prevent neurodegeneration? Autophagy 2006, 302-304. doi: 10.4161/auto.2945

Mizushima, N., Yamamoto, A., Hatano, M., Kobayashi, Y., Kabeya, Y., Suzuki, K., et al. (2001). Dissection of autophagosome formation using Apg5-deficient mouse embryonic stem cells. J. Cell Biol. 152, 657-668. doi: $10.1083 /$ jcb.152.4.657

Mizushima, N., Yoshimori, T., and Levine, B. (2010). Methods in mammalian autophagy research. Cell 140, 313-326. doi: 10.1016/j.cell.2010.01.028

Nader, M. A., Ateyya, H., El-Shafey, M., and El-Sherbeeny, N. A. (2018), Sitagliptin enhances the neuroprotective effect of pregabalin against pentylenetetrazole-induced acute epileptogenesis in mice: implication of oxidative, inflammatory, apoptotic and autophagy pathways. Neurochem. Int. 115, 11-23. doi: 10.1016/j.neuint.2017.10.006

Nakashima, A., Aoki, A., Kusabiraki, T., Shima, T., Yoshino, O., Cheng, S. B., et al. (2017). Role of autophagy in oocytogenesis, embryogenesis, implantation, and pathophysiology of pre-eclampsia. J. Obstet. Gynaecol. Res. 43, 633-643. doi: $10.1111 /$ jog. 13292

Pankiv, S., Clausen, T. H., Lamark, T., Brech, A., Bruun, J. A., Outzen, H., et al. (2007). p62/SQSTM1 binds directly to Atg8/LC3 to facilitate degradation of ubiquitinated protein aggregates by autophagy. J. Biol. Chem. 282, 24131-24145. doi: 10.1074/jbc.M702824200

Poulose, S. M., Fisher, D. R., Bielinski, D. F., Gomes, S. M., Rimando, A. M., Schauss, A. G., et al. (2014). Restoration of stressor-induced calcium dysregulation and autophagy inhibition by polyphenol-rich acai (Euterpe spp.) fruit pulp extracts in rodent brain cells in vitro. Nutrition 30, 853-862. doi: 10.1016/j.nut.2013.11.011

Prock, T. L., and Miranda, R. C. (2007). Embryonic cerebral cortical progenitors are resistant to apoptosis, but increase expression of suicide receptor DISCcomplex genes and suppress autophagy following ethanol exposure. Alcohol. Clin. Exp. Res. 31, 694-703. doi: 10.1111/j.1530-0277.2007.00354.x

Radonjić, N. V., Petronijević, N. D., Vucković, S. M., Prostran, M. S., Nesić, Z. I., Todorović, V. R., et al. (2008). Baseline temperature in an animal model of schizophrenia: long-term effects of perinatal phencyclidine administration. Physiol. Behav. 93, 437-443. doi: 10.1016/j.physbeh.2007.10.003

Rocha-Ferreira, E., and Hristova, M. (2016). Plasticity in the neonatal brain following hypoxic-ischaemic injury. Neural Plast. 2016:4901014. doi: $10.1155 / 2016 / 4901014$

Roos, S., Lagerlöf, O., Wennergren, M., Powell, T. L., and Jansson, T. (2009). Regulation of amino acid transporters by glucose and growth factors in cultured primary human trophoblast cells is mediated by mTOR signaling. Am. J. Physiol. Cell Physiol. 297, C723-C731. doi: 10.1152/ajpcell.00191. 2009

Saribas, A. S., Khalili, K., and Sariyer, I. , K. (2015). Dysregulation of autophagy by HIV-1 Nef in human astrocytes. Cell Cycle 14, 2899-2904. doi: 10.1080/15384101.2015.1069927

Shvets, E., Fass, E., and Elazar, Z. (2008). Utilizing flow cytometry to monitor autophagy in living mammalian cells. Autophagy 4, 621-628. doi: 10.4161/auto.5939 
Song, B., Zhou, T., Liu, J., and Shao, L. (2016). Involvement of programmed cell death in neurotoxicity of metallic nanoparticles: recent advances and future perspectives. Nanoscale Res. Lett. 11:484. doi: 10.1186/s11671-0161704-2

Sun, W., Du, L., Tang, W., Kuang, L., Du, P., Chen, J., et al. (2017). PBDE-209 exposure damages learning and memory ability in rats potentially through increased autophagy and apoptosis in the hippocampus neuron. Environ. Toxicol. Pharmacol. 50, 151-158. doi: 10.1016/j.etap.2017.02.006

Takarada, T., Nakamichi, N., Nakazato, R., Kakuda, T., Kokubo, H., Ikeno, S., et al. (2015). Possible activation by the green tea amino acid theanine of mammalian target of rapamycin signaling in undifferentiated neural progenitor cells in vitro. Biochem. Biophys. Rep. 5, 89-95. doi: 10.1016/j.bbrep.2015.09.021

Tanner, C. M., Kamel, F., Ross, G. W., Hoppin, J. A., Goldman, S. M., Korell, M., et al. (2011). Rotenone, paraquat, and Parkinson's disease. Environ. Health Perspect. 119, 866-872. doi: 10.1289/ehp.1002839

Tekirdag, K. A., and Cuervo, A. M. (2018). Chaperone-mediated autophagy and endosomal microautophagy: joint by a chaperone. J. Biol. Chem. 293, 5414-5424. doi: 10.1074/jbc.R117.818237

Tian, X., Ma, S., Wang, Y., Hou, L., Shi, Y., Yao, M., et al. (2016). Effects of placental ischemia are attenuated by 1,25-dihydroxyvitamin D treatment and associated with reduced apoptosis and increased autophagy. DNA Cell Biol. 35, 59-70. doi: 10.1089/dna.2015.2885

Tseng, T. C., Hsieh, F. Y., and Hsu, S. H. (2016). Increased cell survival of cells exposed to superparamagnetic iron oxide nanoparticles through biomaterial substrate-induced autophagy. Biomater. Sci. 4, 670-677. doi: 10.1039/C5BM00573F

Wang, F., Xu, C., Reece, E. A., Li, X., Wu, Y., Harman, C., et al. (2017). Protein kinase $\mathrm{C}$-alpha suppresses autophagy and induces neural tube defects via miR-129-2 in diabetic pregnancy. Nat. Commun. 8:15182. doi: $10.1038 /$ ncomms15182

Wang, P., Guan, Y. F., Du, H., Zhai, Q. W., Su, D. F., and Miao, C. Y. (2012). Induction of autophagy contributes to the neuroprotection of nicotinamide phosphoribosyltransferase in cerebral ischemia. Autophagy 8, 77-87. doi: 10.4161/auto.8.1.18274

Wang, X. Y., Li, S., Wang, G., Ma, Z. L., Chuai, M., Cao, L., et al. (2015). High glucose environment inhibits cranial neural crest survival by activating excessive autophagy in the chick embryo. Sci. Rep. 5:18321. doi: 10.1038/srep18321

Wen, Y. D., Sheng, R., Zhang, L. S., Han, R., Zhang, X., Zhang, X. D., et al. (2008). Neuronal injury in rat model of permanent focal cerebral ischemia is associated with activation of autophagic and lysosomal pathways. Autophagy 4, 762-769. doi: 10.4161/auto.6412

Xing, S., Zhang, Y., Li, J., Zhang, J., Li, Y., Dang, C., et al. (2012). Beclin 1 knockdown inhibits autophagic activation and prevents the secondary neurodegenerative damage in the ipsilateral thalamus following focal cerebral infarction. Autophagy 8, 63-76. doi: 10.4161/auto.8.1.18217

Yang, F., and Luo, J. (2015). Endoplasmic reticulum stress and ethanol neurotoxicity. Biomolecules 5, 2538-2553. doi: 10.3390/biom5042538

Yoshii, S. R., and Mizushima, N. (2017). Monitoring and measuring autophagy. Int. J. Mol. Sci. 18:1865. doi: 10.3390/ijms18091865

Zhang, F., Zhao, S., Yan, W., Xia, Y., Chen, X., Wang, W., et al. (2016). Branched chain amino acids cause liver injury in obese/diabetic mice by promoting adipocyte lipolysis and inhibiting hepatic autophagy. EBioMedicine 13, 157-167. doi: 10.1016/j.ebiom.2016.10.013

Zhang, Y., Yin, J., Zhang, L., Qi, C. C., Ma, Z. L., Gao, L. P., et al. (2017). Spermidine preconditioning ameliorates laurate-induced brain injury by maintaining mitochondrial stability. Neurol. Res. 39, 248-258. doi: 10.1080/01616412.2017.1283830

Zhang, Z. W., Li, Z. L., and Yuan, S. (2016). The role of secretory autophagy in zika virus transfer through the placental barrier. Front. Cell Infect. Microbiol. 6:206. doi: 10.3389/fcimb.2016.00206

Zhao, Y., Huang, Q., Yang, J., Lou, M., Wang, A., Dong, J., et al. (2010). Autophagy impairment inhibits differentiation of glioma stem/progenitor cells. Brain Res. 1313, 250-258. doi: 10.1016/j.brainres.2009.12.004

Zhao, L. N., Yan, M. L., Wang, X. J., Dou, T. T., Chang, X. L., and Zhou, Z. J. (2016). The effect of paraquat on autophagy in human embryonic neural progenitor cells. Zhonghua Lao Dong Wei Sheng Zhi Ye Bing Za Zhi. 34, 178-183. doi: 10.3760/cma.j.issn.1001-9391

Conflict of Interest Statement: The authors declare that the research was conducted in the absence of any commercial or financial relationships that could be construed as a potential conflict of interest.

Copyright (c) 2018 Lei, Calvo, Vigh and Burd. This is an open-access article distributed under the terms of the Creative Commons Attribution License (CC $B Y)$. The use, distribution or reproduction in other forums is permitted, provided the original author(s) and the copyright owner are credited and that the original publication in this journal is cited, in accordance with accepted academic practice. No use, distribution or reproduction is permitted which does not comply with these terms. 